\title{
Bibliotecas digitales y los centros de información
}

\section{Digital libraries and information centers}

\author{
Erika Aurora Castillo Celis \\ Ruth Noemy Ruíz Mangandi \\ Zoila Lidia Chó Chiguichón \\ Olga Beatriz Xicará García \\ Claudina Aifan Pineda \\ Herwer Orlando Castillo Valdés \\ Carlos Humberto Chiroy \\ Olga Yolanda Marroquín Espinoza \\ Universidad de San Carlos de Guatemala \\ https://orcid.org/0000-0001-9658-655X
} Maestría en Bibliotecología y Ciencia de la Información Correo para recibir correspondencia: erikaccelis@gmail.com

\section{$\triangle$ Referencia del artículo}

Castillo-Celis, E. A., Ruíz-Mangandi, R. N., Chó-Chiguichón, Z. L. Xicará-García, O. B., Aifan-Pineda, C., Castillo-Valdés, H. O., Chiroy, C. H., Marroquín-Espinoza, O. Y (2001). Bibliotecas digitales y los centros de información. Revista Guatemalteca de Cultura. 1(1), 1 - 9.

DOI: 10.46954/revistaguatecultura.v1i1.5

Recibido 15/09/ 2020

Aceptado 25/01/2021

\section{Resumen}

El objetivo del presente artículo, brindar una visión panorámica acerca de los servicios de las bibliotecas digitales, entre ellos, la digitalización de documentos, que en los últimos años se convirtió en una actividad esencial que facilitó el acceso a los recursos digitales. La metodología utilizada en la redacción de este artículo es documental, lo que permitió dar lectura a diferentes artículos científicos, escritos por expertos en el campo de la bibliotecología y la tecnología. 
La tecnología ha revolucionado las formas de comunicar la información, por lo que las bibliotecas se han visto beneficiadas. Este trabajo pretende enfatizar que la participación del bibliotecario en la educación y sociedad actual, es vital para el procesamiento y socialización de la información, para que exista acceso al conocimiento.

Palabras clave: bibliotecas digitales, bibliotecas innovadoras, bibliotecas públicas

\section{Abstract}

The objective of this article is to provide the reader with a panoramic view of the services of digital libraries, including the digitization of documents, which in recent years has become an essential activity that facilitated access to digital resources. The methodology used in the writing of this article is documentary, which made it possible to read several scientific articles written by experts, which are freely accessible. Technology has revolutionized the forms of information communication, which is why libraries have been benefited. This is how this work aims to show that the participation of the librarian in education and in society is vital for the processing and socialization of information so that everyone has access to knowledge.

< Keywords: digital libraries, innovative libraries

\section{Introducción}

Desde sus inicios, las bibliotecas han evolucionado la forma de transmitir el conocimiento, adaptándose fielmente a las necesidades de los usuarios, reorientando así su misión, visión, servicios y todo lo que les permita estar a la altura de la curiosidad y evolución humana. Tras el impulso de las Digital Libraries Initiatives I y II norteamericanas y el soporte del Digital Libraries Program de la Unión
Europea, en la década de los noventa, se han visto cambios que han marcado su evolución. Se mencionan también, los problemas que influyen en la transformación de la biblioteca convencional.

La revolución tecnológica y el uso de las TIC's en las bibliotecas, requiere del empoderamiento de profesionales con habilidades y competencias para saber utilizar, analizar, procesar (proceso técnico y digitalización), comunicar y transmitir 
la información que se encuentra en diferentes formatos y soportes. Uso del software, aplicaciones, manejo de equipos disponibles en el mercado, que a la vez son tan cambiantes. Por último, es de suma importancia mencionar que el profesional de la información, debe de conocer la legislación sobre todo en cuanto a derechos de autor, antes de su publicación digital.

El presente estudio permite tener una visión más amplia de la tecnología en las bibliotecas y centros de información, dar a conocer un mundo de posibilidades para acceder al conocimiento y lograr un mejor funcionamiento, así como para ofrecer e intercambiar información. Cada vez son más las bibliotecas que implementan cambios en sus actividades, en su infraestructura física y tecnológica, para convertirse en centros culturales que van más allá de los servicios tradicionales, en donde el usuario encuentra diversidad de actividades que realizar, su esparcimiento, negocios, trabajo entre otros beneficios que ésta pueda brindar.

Un ejemplo de ello es la biblioteca de Chattanooga, Tennessee, la cual ha recibido el reconocimiento externo en relación a sus servicios, pues conserva los servicios tradicionales de biblioteca, pero también ofrece servicios de impresión 3D promoviendo la cultura maker. Como lo expresa AlonsoArévalo y Cordón-García (2015) Ofrece clases de programación de ordenadores, vídeo y software de audio-producción. Recursos de avance que para cualquier persona son demasiado caros o difícil acceso, los puede encontrar y beneficiarse del uso en este espacio comunitario (p. 7).
La revolución tecnológica y el uso de las TIC's en las bibliotecas, requiere del empoderamiento de profesionales de la información con habilidades y competencias propias del campo bibliotecológico, digitalización, y comunicación para luego transmitir la información que se encuentra en diferentes formatos y soportes, sin dejar de mencionar el uso del software, aplicaciones, manejo de equipos disponibles en el mercado que a la vez son tan cambiantes. Por último, es sumamente importante recalcar que es el profesional de la información el encargado de hacer valer la legislación en cuanto a los derechos de reproducción, al momento de digitalizar la información de la biblioteca.

\section{Biblioteca pública digital}

UNESCO (1994), declara en su Manifiesto para Bibliotecas Públicas que:

"la finalidad de la biblioteca es facilitar recursos informativos y prestar servicios mediante diversos medios con el fin de cubrir las necesidades de personas y grupos en materia de instrucción, información y perfeccionamiento personal comprendidas actividades intelectuales de entretenimiento y ocio, pues desempeñan un importante papel en el progreso y el mantenimiento de una sociedad democrática al ofrecer a cada persona acceso a toda una serie de conocimientos, ideas y opiniones" (p. 8 )

en 2001 reitera sus directrices afirmando que la biblioteca:

"Brinda acceso al conocimiento, la información y las obras de la imaginación gracias a toda una serie de recursos y 
servicios y está a disposición de todos los miembros de la comunidad por igual, sean cuales fueren su raza, nacionalidad, edad, sexo, religión, idioma, discapacidad, condición económica y laboral y nivel de instrucción" (p. 8).

De acuerdo a las necesidades del ser humano moderno, la biblioteca no debe quedarse rezagada en los servicios tradicionales, ya que los avances tecnológicos son constantes. Es necesario poner a disposición de la sociedad, una variedad de recursos y servicios tecnológicos que respondan a las necesidades y demandas de todos los usuarios, por lo tanto el bibliotecario deberá poseer las competencias tecnológicas necesarias que todo profesional de la bibliotecología, debe poseer en la actualidad.

La biblioteca que por mucho tiempo ha funcionado de forma tradicional y actualmente debe adaptarse a la era informática actual, poniendo a disposición de la comunidad de usuarios, recursos y servicios electrónicos, que bien es cierto, implican gestiones, gastos y situaciones complicadas, pero con una actitud positiva y una proyección adecuada, la biblioteca dejaría de ser un lugar donde solamente se llega a leer libros. Juntamente con la implementación de nuevos recursos y servicios de biblioteca, se contempla la inserción de diversas actividades que involucren a la comunidad, tales como exposiciones de arte, conferencias presenciales con ponentes expertos, tanto nacionales como extranjeros, a través de plataformas digitales como Zoom, Google Meet o Microsoft Team, que aportan beneficios como el acercamiento inmediato con cualquier persona, fomentan la comunicación y el trabajo colaborativo, entre muchas ventajas más.
Hoy en día la biblioteca pública juega un papel fundamental en la educación, ya que puede fomentar y promover la educación a distancia; esto con la implementación de TIC's, como estrategia de aprendizaje. Esto representa una ventaja a largo plazo en relación a reducción de costos, ya que, de esta manera el alcance y proyección sería mayor, alcanzando a un número mayor de usuarios, utilizando métodos de planificación y creación de aulas virtuales, difundiendo información y conocimiento en formatos digitales, entre libros, revistas, folletos, documentales, películas, videos, etc., incluyendo los formatos que requieran el uso de dispositivos móviles como teléfonos celulares, tabletas o computadoras.

El avance tecnológico, ha permitido que los bibliotecarios del mundo puedan trabajar los entornos de información y conocimiento: catalogando, clasificando, indizando y finalmente digitalizando la información de acceso abierto para que los usuarios tengan al alcance de su mano, diversos materiales con los que cuenta la biblioteca. Esta es la función que cumplen día a día los bibliotecólogos actuales, pudiendo así desenvolverse adecuadamente en el entorno legal y profesional, desarrollando en sus comunidades una cultura de respeto hacia los derechos de autor, y permitiendo el acceso de forma eficaz a la información que necesitan, desde el lugar donde se encuentren.

En el proceso de digitalización en las bibliotecas, Voutssás (2006) afirma "Dado que digitalizar un documento, es un proceso de representación fiel del mismo, por un conjunto de números y hay más de una representación posible para cada documento; es decir, pueden establecerse diversas maneras o "patrones" para digitalizar un documento" (p.62). 
En tal contexto de esta afirmación, todo objeto digital está representado con el sistema binario a través de los números 1 y 0 , los expertos explicaron que era el sistema ideal para transmitir toda la información necesaria de un sistema análogo a uno digital y poderse visualizar en los dispositivos electrónicos, en los que se puede mencionar: formato pdf, html, documentos word, dvd, mp3, mp4, jpeg, entre otros; la mayoría de ellos tienen como común denominador que soportan metadatos y cada uno de ellos posee un proceso específico para su digitalización, a través de dispositivos y/o software.

Los documentos digitalizados no son una exclusividad de las bibliotecas digitales, ya que, desde la década de los noventas, se ha experimentado con diferentes formas de preservación, equilibrando así la importancia entre la preservación y resguardo de la información para generaciones futuras y su fácil difusión.

\section{Los cambios en las bibliotecas}

En el libro titulado "Bibliotecas y publicaciones digitales", del autor Juan Voutssás Márquez, da inicio con la historia de la compañía Cyan "edita un juego de computadora llamado Myst" (Cyan Worlds, 2001, p 1) el juego hace referencia a la búsqueda de un libro, donde el personaje lo deja caer en el espacio y el libro se desintegra, posteriormente debe recuperar todas las hojas y reconstruir la información. Este ejemplo es comparado con los usuarios que buscan información diseminada, lo cual lo hace interesante. El juego en mención es una biblioteca, ya que se practican todos los métodos de búsqueda, análisis y síntesis, como en una biblioteca, pero lo que lo hace esto aún más interesante, es que las personas aprenden a navegar en el mundo electrónico.

Asimismo, en el libro, se hace referencia al análisis minucioso de sus partes y el entorno que le rodea. Su interrelación y características desde la perspectiva de su origen, evolución, estado del arte y tendencias de la biblioteca digital; también ofrece información sobre cómo concebir, diseñar y construir una biblioteca digital. Señala las diferencias entre colección digital y material digital o digitalizado, como también la naturaleza y origen de cada definición; orienta sobre la valorización de la información acerca de los materiales implicados en la digitalización, sobre cuándo, cómo y por qué debe digitalizarse tal o cual documento

Por su parte, García Marco (1996) menciona a Vannevar Bush como uno de los precursores del hipertexto afirmando que es uno de los padres de la documentación automatizada, a través de su propuesta de un sistema documental relacional, que es el antecedente directo del hipertexto propuesto por Theodore Nelson, y remoto de la World Wide Web (p.1). Acerca de cómo veía Bush la vida en un futuro tecnificado, en 1945 concibió una máquina para acceder al conocimiento universal. No obstante, esta visión es tan asombrosamente parecida a lo que hoy se observa, a pesar de haber sido escrita hace sesenta años. 
Para brindar una vista general de la evolución de la biblioteca convencional desde sus inicios hasta nuestros días, se menciona que el término biblioteca automatizada surgió en la década de los sesenta, cuando las bibliotecas integraron las primeras computadoras a su quehacer diario. Para que los usuarios tuvieran acceso a los contenidos, se digitaron los índices, resúmenes, directorios, etc. De esta manera, la biblioteca virtual se organizaba para ser alojada en un espacio virtual no físico.

La biblioteca digital nos permite en la actualidad recuperar todo tipo de documentación, e información pormedio de catálogos electrónicos en línea a través de grandes sistemas de gestión de biblioteca, esto sin duda alguna es parte del desarrollo mundial debido a la difusión de la cultura y el conocimiento, convirtiéndose en una herramienta fácil y práctica, que facilita el acceso a la información que el usuario necesita, para luego ser gestionada y utilizada por éste, para la generación de nuevos conocimientos

En el Diccionario en línea de Bibliotecología y Ciencias de la Información ODLIS (2002) se puede apreciar que una biblioteca sin paredes es aquella en la que las colecciones no existen sobre papel, microforma, ni en ninguna forma tangible, pero es electrónicamente accesible en formatos digitales. El problema de definir el concepto comienza con que la biblioteca digital no tiene el mismo significado para distintas comunidades no bibliotecarias. Muchas personas consideran la WWW, como una red que está acumulando muchos documentos y tienden a llamar a esta colección Biblioteca Digital, aunque no fue diseñada para sostener la publicación organizada ni la recuperación de información como lo son las bibliotecas.
Para que la biblioteca digital se pueda analizar correctamente, es necesario examinar el tema desde distintas perspectivas como, la gestión de las colecciones, recursos tecnológicos, tipos de usuarios, organización documental, aspectos legales, culturales, sociopolíticos y económicos. Las bibliotecas digitales están contenidas dentro de Internet y por tanto son parte de los recursos documentales de la red mundial.

\section{Materiales y métodos}

La metodología utilizada en esta investigación fue documental, lo que permitió consultar en Internet y dar lectura a varios artículos científicos, documentación de la UNESCO y libros entre otros, todos de acceso libre, los cuales proporcionaron gran cantidad de información relacionada a la investigación.

\section{Resultados}

Al analizar las innovaciones de bibliotecas digitales y los centros de información, no se puede dejar sin mencionar la digitalización, bibliotecas virtuales, y repositorios documentales. Según Bargiela y colaboradores (2007) la digitalización de documentos es parte fundamental de toda biblioteca virtual y todo centro de documentación como parte esencial en el proceso de aprendizaje a distancia ( $p$. 258). Al utilizar las tecnologías en el campo de la documentación, se pone en práctica diferentes mecanismos que permiten conservar y preservar el patrimonio documental, para su consulta posterior y estas perduren durante el transcurso del tiempo, es por eso que las 
nuevas tecnologías se han convertido en una herramienta indispensable en el ámbito educativo; la revolución de las TIC ha permitido atravesar obstáculos relacionados con el tiempo y el espacio entre las diferentes culturas existentes. En este contexto, la digitalización de documentos es un paso importante e indispensable en la publicación y formulación de servicios en la biblioteca virtual.

Es necesario establecer propuestas que se apeguen a metodologías que suplan las necesidades de información de los usuarios, abordar los cambios que del avance tecnológico trae consigo y cumplir los propósitos de la biblioteca virtual y digital. Una de las desventajas es la pérdida de información, cuando se migra a otro formato o programa, ya que, con el avance tecnológico, día a día aparecen diferentes formas de resguardar la información.

\section{Discusión}

La biblioteca digital debe de contar con diversidad de formatos de texto, gráficos, pdf, audio, videos, html, CDs, DVD, JPG, mp4 almacenadosen un servidor, paraserdistribuidos, además deben de contener diversas temáticas multidisciplinaria o especializada, las cuales deben ser de acceso libre, sin restricciones, sin permanecer en los anaqueles, como están las obras físicas. Una biblioteca digital debe de contar con un sistema de tratamiento técnico, acceso y transferencia de información digital, estructurado alrededor del ciclo de vida de una colección de documentos digitales, sobre de los cuales se ofrecen servicios interactivos de valor agregado para el usuario final.
Lo adecuado es cooperar con la biblioteca universal, para visualizar la producción de conocimiento autóctono, étnico propios de los contextos culturales de su lugar o región y los informes científicos que surjan. Todo lo visualizado a través de las bibliotecas debería ser acceso libre para facilitar el acceso a la información a los usuarios. La era digital ha abierto grandes oportunidades de aprendizaje y el desarrollo de la información; lo importante es la formación y actualización en el ámbito tecnológico, estar alfabetizados informacionalmente, adquirir habilidades y competencias que permitan a los profesionales de la información, estar a la altura de las necesidades y requerimientos de los usuarios, garantizando de esta manera, un mejor servicio. 


\section{Referencias}

Alonso Arevalo, J., Cordón García, A. (2015). ¿Para qué servirá la Biblioteca Pública en el futuro? Depende de su capacidad de adaptación a los imparables cambios sociales, económicos y tecnológicos.? DBD. Artículos del Departamento de Biblioteconomía y Documentación [290, s.n] Disponible en: https:// gredos.usal.es/bitstream/handle/10366/125206/MiBibliotecaNNTTMB401.pdf?sequence $=1$

Bargiela, R., Dapozo, G. N., \& Bogado, V. S. (2007). Digitalización de documentos para su utilización en una biblioteca virtual. In II Congreso de Tecnología en Educación y Educación en Tecnología. Disponible en: http://sedici.unlp.edu.ar/bitstream/ handle/10915/19150/31.pdf?sequence $=1$

Cyan Worlds (2001). Disponible en: http://www. cyan.com

García Marco, F. J. (1996). Vannevar Bush, el hipertexto y el futuro del documento. TENDENCIAS DE INVESTIGACIÓN EN DOCUMENTACIÓN, Zaragoza: Jesús Tramullas (editor), 185-209. https://www.researchgate.net/publication/304627708_Vannevar_ Bush_el_hipertexto_y_el_futuro_del_documento

Márquez, J. V. (2006). Bibliotecas y publicaciones digitales. UNAM. Disponible en: https://libros.metabiblioteca.org/jspui/ bitstream/001/345/4/970-32-3962-5.pdf

ODLIS On-Line Dictionary of Library and Information Science. (2002) Western Connecticut State University. Disponible en en: http:// www.wcsu.edu/library/ odlis.html

UNESCO (2001) Directrices IFLA/UNESCO para el desarrollo del servicio de bibliotecas públicas. Disponible en: https://www.ifla.org/files/ assets/hq/publications/archive/the-public-libra

UNESCO (2008) Directrices IFLA/UNESCO para el desarrollo del servicio de bibliotecas públicas. Disponible en: https://www.ifla.org/files/ assets/hq/publications/archive/the-public-library-service/pg01-s.pdf

Voutssás, J. (2006). Bibliotecas y publicaciones digitales. Universidad

Nacional Autónoma de México. Disponible en: https://libros. metabiblioteca.org/jspui/bitstream/001/345/4/970-32-3962-5.pdf 


\section{Sobre los autores}

Equipo de investigadores de la Maestría en Bibliotecología y Ciencia de la Información de la Universidad de San Carlos de Guatemala.

Copyright (c) Erika Autora Castillo Celis y colaboradores

Este texto está protegido por una licencia Creative Commons 4.0.
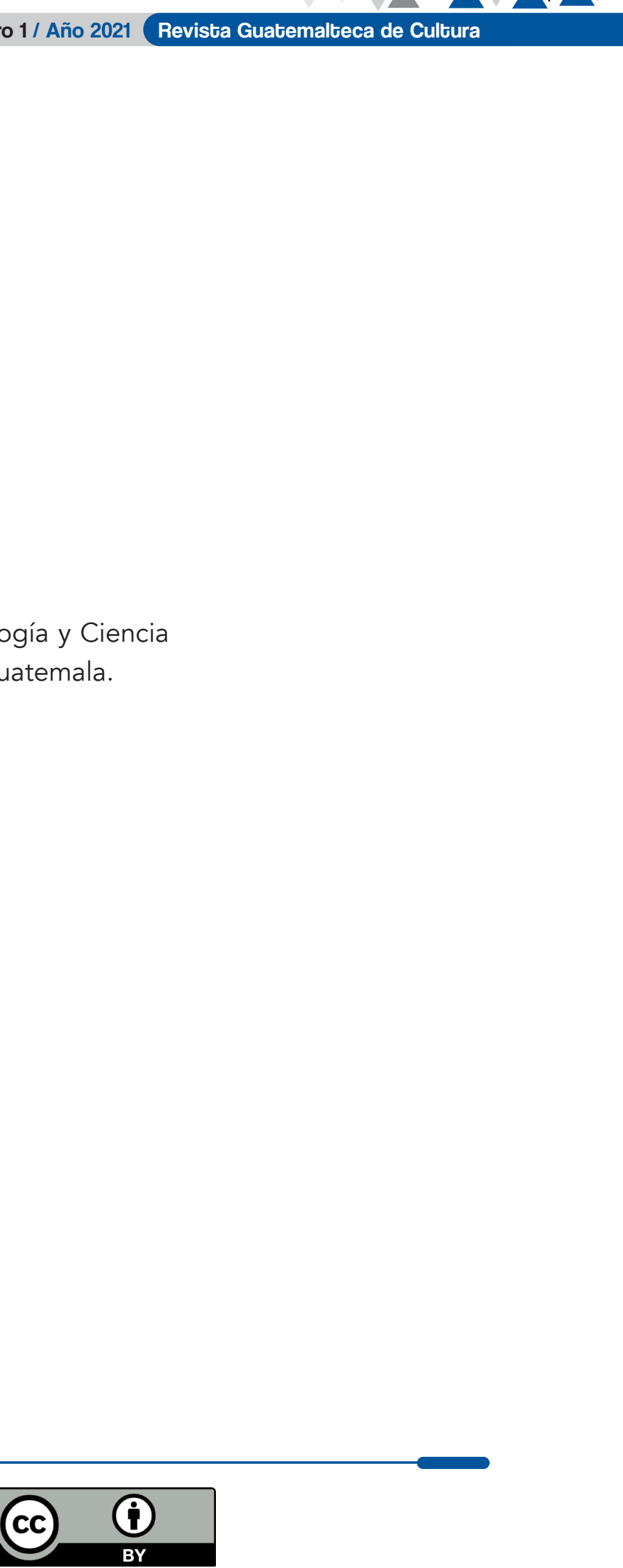

Usted es libre para compartir, copiar y redistribuir el material en cualquier medio o formato y adaptar el documento, remezclar, transformar y crear a partir del material para cualquier propósito, incluso comercialmente, siempre que cumpla la condición de atribución: usted debe reconocer el crédito de una obra de manera adecuada, proporcionar un enlace a la licencia, e indicar si se han realizado cambios. Puede hacerlo en cualquier forma razonable, pero no de forma tal que sugiera que tiene el apoyo del licenciante o lo recibe por el uso que hace. 